\title{
RESPOSTAS FISIOLÓGICAS E GRADIENTES TÉRMICOS DE OVINOS DAS RAÇAS SANTA INÊS, MORADA NOVA E DE SEUS CRUZAMENTOS COM A RAÇA DORPER ÀS CONDIÇÕES DO SEMI-ÁRIDO NORDESTINO ${ }^{1}$
}

\author{
Physiologic responses and thermal variation of Santa Inês, Morada Nova sheep and their \\ crossbreed with Dorper breed to the semi-arid northeastern of Brazil
}

\author{
Jose Romulo Soares dos Santos ${ }^{2}$, Bonifácio Benício de Souza ${ }^{3}$, Wandrick Hauss de Souza ${ }^{4}$, \\ Marcilio Fontes Cezar ${ }^{3}$, Gustavo de Paula Tavares ${ }^{5}$
}

\begin{abstract}
RESUMO
Este trabalho foi desenvolvido na Empresa Estadual de Pesquisa Agropecuária da Paraíba S.A. (EMEPA-PB), no município de Soledade, com o objetivo de avaliar os parâmetros fisiológicos (frequiência respiratória, freqüência cardíaca e temperatura retal) e gradientes térmicos (entre temperatura retal e temperatura da superfície e entre temperatura da superfície e temperatura do ar) de ovinos das raças Santa Inês, Morada Nova e seus mestiços F1 com ovinos Dorper, no semi-árido nordestino. Foram utilizados 30 ovinos, de cinco genótipos diferentes, distribuídos num delineamento inteiramente casualizado, num esquema fatorial 5 x 2; cinco genótipos (Santa Inês, Morada Nova, $1 / 2$ Santa Inês $+1 / 2$ Dorper, $1 / 2$ Santa Inês $+1 / 2$ Morada Nova, 1/2 Morada Nova + 1/2 Dorper) vs dois turnos (manhã e tarde), com 6 repetições. Houve interação entre genótipo e turno. Detectou-se efeito significativo $(\mathrm{P}<0,05)$ entre genótipos apenas para as variáveis freqüência cardíaca e gradiente entre temperatura superficial e temperatura do meio, no turno da manhã. O turno exerceu efeito significativo $(\mathrm{P}<0,05)$ sobre todos os parâmetros fisiológicos analisados. Concluiu-se que os ovinos das raças Santa Inês, Morada Nova e seus mestiços com a raça Dorper apresentam alto grau de adaptabilidade, às condições Semi-áridas do Brasil.
\end{abstract}

Termos para indexação: Bioclimatologia, estresse térmico, genótipos, parâmetros fisiológicos.

\begin{abstract}
This work was conducted in the State Company of Agricultural Research of Paraíba S.A. (EMEPA-PB), in the district of Soledade, with the objective of evaluating the physiologic parameters (breathing frequency, heart frequency and rectal temperature) and thermal variation (between rectal and skin temperature and between skin and air temperature) of Santa Inês and Morada Nova sheep and their crossbreeds F1 with the Dorper sheep, under to the climatic conditions of the semi-arid Northeastern. Thirty sheep were used, of five different genotypes, distributed in a random customized design, in a factorial outline $5 \mathrm{x} 2$; five genotypes (Santa Inês, Morada Nova, 1/2Santa Inês + 1/2Dorper, 1/2Santa Inês + 1/2Morada Nova, 1/2Morada Nova + 1/2Dorper) vs two shifts (morning and afternoon), with 6 repetitions. Interaction was detected among variables genotype and shift. There was significant effect $(\mathrm{P}<0,05)$ among genotypes only on the variables heart frequency and thermal variation between skin and air temperature, in the shift of the morning. The shift influenced significantly $(\mathrm{P}<0,05)$ all the analyzed physiologic parameters. It is concluded that Santa Inês, and Morada Nova sheep and their crossbreeds with the Dorper sheep showed to have high adaptability in the semi-arid conditions of Brazil.
\end{abstract}

Index terms: Adaptability, heat stress, genetic groups, physiologic responses.

(Recebido para publicação em 4 de novembro de 2004 e aprovado em 2 de março de 2006)

\section{INTRODUÇÃO}

Uma das estratégias para aumentar a produtividade dos rebanhos ovinos, no semi-árido, tem sido a introdução de raças sul-africanas, como a Dorper, para serem utilizadas em cruzamentos com raças nativas. O meio ambiente tem influência em muitos aspectos da produção animal e o sucesso de uma criação depende da escolha de raças ou produtos de cruzamentos que sejam melhor adaptados às condições climáticas de uma determinada região.
A produtividade animal depende da capacidade do animal manter a temperatura corporal. Os animais utilizam vários mecanismos para manter a homeotermia, como a vasodilatação periférica (CHIMINEAU, 1993). Segundo Habeeb et al. (1992), o redirecionamento do fluxo sangüíneo para a superfície corporal, pela vasodilatação, aumenta a temperatura da superfície do animal facilitando a dissipação de calor por mecanismos não evaporativos (condução, convecção e radiação). Entretanto, a eficácia desses mecanismos depende do gradiente térmico entre o corpo

\footnotetext{
${ }^{1}$ Pesquisa autor financiada pelo Banco do Nordeste e CNPq.

2 Estudante do curso de Medicina Veterinaria, bolsista de Iniciação Científica PIBIC/CNPQ - UFCG/CSTR/DMV - Cx. P. 64 - 58.700-000 - Patos, PB jromulosmedicoveterinario@bol.com.br

3 Professor da Universidade Federal de Campina Grande/UFCG - DMV - Cx. P. 64 - 58.700-000 - Patos, PB - bonif@cstr.ufcg.edu.br

${ }^{4}$ Pesquisador da EMEPA, João Pessoa, PB.

${ }^{5}$ Estudante de Medicina Veterinária da Universidade Federal de Campina Grande/UFCG - Cx. P. 64 - 58.700-000 - Patos, PB.
} 
do animal e o ambiente. Quanto maior o gradiente maior será a dissipação de calor. A pele mais quente do animal tende a perder calor em contato com o ar mais frio. Se a temperatura do ar aumenta, diminui essa perda de calor por meio do calor sensível, aumentando a temperatura do núcleo central; daí o organismo animal, através de mecanismos evaporativos, como a sudorese e/ou freqüência respiratória, aumenta a dissipação de calor insensível. A forma insensível de dissipação de calor é regulada pela umidade, ou seja, quanto maior a umidade mais será comprometido esse mecanismo de dissipação. Dessa forma, a temperatura do ar e a umidade são consideradas como os principais elementos climáticos responsáveis pelo incremento calórico à temperatura corporal dos animais (DOMINGUES, 1968; HARDY, 1981; HARRIS et al., 1960; SHORODE et al., 1960). Se o animal não conseguir dissipar calor excedente através dos mecanismos citados, a temperatura retal aumenta acima dos valores fisiológicos normais e desenvolve-se o estresse calórico, responsável em parte pela baixa produtividade animal nos trópicos.

Para Olivier (2000), a avaliação de uma raça ou grupo genético não pode ser baseada apenas na capacidade de ganho de peso e no rendimento de carcaça, mas também na eficiência produtiva, adaptabilidade, prolificidade e taxa de sobrevivência. Assim, se a performance produtiva for baseada apenas no desempenho produtivo, pode estar sujeita a erros. Para Abi-Saab \& Sleiman (1995), os critérios de tolerância, longevidade e adaptação dos animais são determinados pelas medidas fisiológicas da respiração, freqüência cardíaca e temperatura corporal. Para BrownBrandl et al. (2003), a temperatura retal é um bom indicador do estresse térmico. Segundo Bianca \& Kunz (1978), a temperatura retal e a frequiência respiratória são consideradas as melhores variáveis fisiológicas para estimar a tolerância de animais ao calor e em menor escala tem sido objeto de estudo a freqüência cardíaca (FC), temperatura da pele $(\mathrm{Tp})$ e os constituintes sangüíneos (SILVA \& GONDIM, 1971). Com relação à freqüência cardíaca (FC), Silva \& Gondim (1971) reportam que essa é uma variável sujeita a um grande número de fatores além da temperatura ambiente, como a idade, a individualidade, o temperamento e o grau de excitação do animal.

O estudo bioclimatológico sobre a introdução de novas raças ou produtos de cruzamento, na região semiárida, é importante para a determinação de genótipos mais adequados à condição ambiental específica do semi-árido, para se obter uma melhor produtividade (MONTY JUNIOR et al., 1991). Assim, objetivou-se com este trabalho, avaliar a adaptabilidade fisiológica de ovinos Santa Inês, Morada Nova e seus mestiços (produtos F1) entre as duas raças e com a raça Dorper, às condições climáticas do trópico semiárido nordestino.

\section{MATERIAL E MÉTODOS}

O experimento foi conduzido na Empresa Estadual de Pesquisa Agropecuária da Paraíba S.A. (EMEPA), localizada no município de Soledade, Microrregião do Curimataú ocidental do Agreste paraibano, situada a $7^{\circ} 8^{\prime} 18^{\prime \prime}$ 'S e $36^{\circ} 27^{\prime} 2^{\prime \prime}$ W. Gr. e a uma altitude em torno de $534 \mathrm{~m}$. Essa região caracteriza-se por clima do tipo

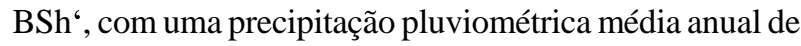
$500 \mathrm{~mm}$. A média da temperatura máxima anual é de $35^{\circ} \mathrm{C}$ e a mínima é de $22^{\circ} \mathrm{C}$, com pequenas variações. A umidade relativa do ar situa-se em torno de $50 \%$.

Foram utilizados 30 cordeiros machos castrados, com peso vivo médio de $18-20 \mathrm{~kg}$,; distribuídos num delineamento inteiramente casualizado, num esquema fatorial 5 x 2; cinco genótipos (Santa Inês, Morada Nova, $1 / 2$ Santa Inês $+1 / 2$ Dorper, $1 / 2$ Santa Inês $+1 / 2$ Morada Nova, $1 / 2$ Morada Nova $+1 / 2$ Dorper) vs dois turnos (manhã e tarde), com 6 repetições. Os animais ficaram confinados em galpão, alojados em gaiolas de madeira, sendo a dieta composta por feno de maniçoba (Manihot glaziowii Mull. Arg.), forrageira nativa da caatinga, mais uma mistura concentrada (milho, soja, óleo vegetal e sal mineral). A ração concentrada foi formulada segundo AFRC (1995) e ARC (1980), para um ganho de peso médio diário de $200 \mathrm{~g}$.

Foram instalados no ambiente experimental um termohigrógrafo, um termômetro de máxima e mínima; um termômetro de globo negro, termômetros de bulbo seco e um bulbo úmido. Com objetivo de medir e registrar a temperatura e a umidade relativa do ar, máxima e mínima, temperatura de globo negro (TGN), temperatura do ponto de orvalho (Tpo) e determinação do índice de temperatura de globo negro e umidade (ITGU), durante o período experimental. Segundo Buffington et al. (1981), o cálculo do ITGU obedece a seguinte fórmula: ITGU $=\mathrm{TGN}+$ $0,36 *(\mathrm{Tpo})+41,5$. As leituras das variáveis ambientais foram feitas às 9:00 e 15:00 horas, de acordo com Brasil (1992).

Os parâmetros estudados foram: temperatura retal (TR), determinada através da introdução de um termômetro clínico veterinário, com escala até $44{ }^{\circ} \mathrm{C}$, diretamente no reto do animal, permanecendo por um período de um minuto, em seguida retirado e o resultado da leitura expresso em graus centígrados; frequiência respiratória (FR), determinada através da auscultação indireta das bulhas, com auxílio de estetoscópio flexível, ao nível da região 
laringo-traqueal e expressa em movimentos por minuto; frequiência cardíaca (FC) obtida com o auxílio de um estetoscópio flexível, colocado diretamente na região torácica esquerda à altura do arco aórtico e expressa em batimentos por minuto (BACARI JÚNIOR, 1990); gradientes térmicos (entre temperatura interna-temperatura superficial do animal ("TR-TS) e entre temperatura superficialtemperatura ambiente ("TS-TA)). As coletas de dados ocorreram nos turnos da manhã (9:00 horas) e da tarde (15:00 horas), dois dias por semana, durante quatro semanas, na época quente e seca do semi-árido nordestino brasileiro.

Os resultados obtidos foram submetidos à análise de variância pelo programa estatístico SAS Institute (1996) e os valores médios foram comparados pelo teste de Tukey a $5 \%$ de probabilidade.

\section{RESULTADOS E DISCUSSÃO}

As médias dos dados meteorológicos, observadas durante a fase experimental, encontram-se na Tabela 1.

Os valores de ITGU observados na TABELA 1, 70 pela manhã e 79 pela tarde representam respectivamente, ambientes de conforto térmico e ambiente perigoso, de acordo com estudo realizado com bovinos pelo National Weather Service-Central Region, citado por Baêta \& Souza (1997).

As médias da temperatura retal, freqüências respiratória e cardíaca dos genótipos encontram-se na Tabela 2.

A avaliação da temperatura retal (TR) permitiu observar que houve interação entre genótipo e turno. Tendo o mestiço $1 / 2$ Santa Inês $+1 / 2$ Dorper diferido estatisticamente $(\mathrm{P}<0,05)$ de todos os genótipos no turno da manhã apresentando maior TR $\left(39,66^{\circ} \mathrm{C}\right)$. À tarde, o mestiço $1 / 2$ Santa Inês $+1 / 2$ Dorper diferiu apenas dos genótipos puros, tendo também apresentado maior TR $\left(39,72^{\circ} \mathrm{C}\right)$. O genótipo Morada Nova, no turno da manhã, apresentou menor $\operatorname{TR}\left(39,02^{\circ} \mathrm{C}\right)$ diferindo estatisticamente $(\mathrm{P}<0,05)$ apenas dos mestiços $1 / 2$ Santa Inês $+1 / 2$ Dorper e $1 / 2$ Morada Nova $+1 / 2$ Santa Inês. À tarde também apresentou menor TR $\left(39,24^{\circ} \mathrm{C}\right)$ diferindo estatisticamente $(\mathrm{P}<0,05)$ dos demais genótipos. Os demais genótipos não diferiram entre si $(\mathrm{P}>0,05)$ independentemente de turno. Embora possa haver diferença significativa entre genótipos, entre turnos e dentro de cada turno, todos os animais mantiveram a temperatura retal na média, mantendo a homeotermia, de acordo com Swenson \& Reece (1996).

Houve significância de turno $(\mathrm{P}<0,05)$ apenas para o $1 / 2$ Morada Nova $+1 / 2$ Dorper. Arruda, citado por Souza et al. (1990), considera uma maior elevação da temperatura retal no turno da tarde como sendo um índice de menor adaptabilidade, dessa forma apenas o mestiço $1 / 2$ Morada Nova $+1 / 2$ Dorper seria considerado o menos adaptado. Souza et al. (1990) verificaram que a raça Morada Nova apresentou maior temperatura retal à tarde, em relação à da manhã, do que o Santa Inês, o que difere dos resultados encontrados neste trabalho. Souza et al. (1990) relatam que a temperatura retal da raça Santa Inês, pela manhã, foi superior à da raça Morada Nova, invertendo-se no turno da tarde, o que difere dos resultados observados neste trabalho apenas no turno vespertino. As temperaturas retais médias, observadas neste experimento, foram superiores as registradas por Souza et al. (1990) para as raças Santa Inês e Morada Nova $\left(38,73\right.$ e $38,72^{\circ} \mathrm{C}$, respectivamente); isto se deve provavelmente à alta umidade que pode ter dificultado a dissipação de calor observada no presente trabalho.

TABELA 1 - Valores médios da temperatura do bulbo seco (TBS), bulbo úmido (TBU), termômetro de globo negro (TGN), umidade relativa (UR), temperatura máxima (TMX) e mínima (TMN), temperatura do ponto de orvalho (Tpo) e índice de temperatura do globo negro e umidade (ITGU) nos turnos da manhã e da tarde.

\begin{tabular}{|c|c|c|}
\hline \multirow{2}{*}{ Variáveis } & \multicolumn{2}{|c|}{ Turnos } \\
\hline & Manhã & Tarde \\
\hline $\operatorname{TBS}\left({ }^{\circ} \mathrm{C}\right)$ & 20 & 28 \\
\hline $\operatorname{TBU}\left({ }^{\circ} \mathrm{C}\right)$ & 17 & 19 \\
\hline $\mathrm{TGN}\left({ }^{\circ} \mathrm{C}\right)$ & 23 & 32 \\
\hline UR (\%) & 74 & 42 \\
\hline Tpo $\left({ }^{\circ} \mathrm{C}\right)$ & 15,2 & 13,9 \\
\hline ITGU & 70 & 79 \\
\hline $\mathrm{TMX}\left({ }^{\circ} \mathrm{C}\right)$ & \multicolumn{2}{|c|}{30} \\
\hline $\operatorname{TMN}\left({ }^{\circ} \mathrm{C}\right)$ & \multicolumn{2}{|c|}{19} \\
\hline
\end{tabular}


A temperatura retal é o resultado entre a energia térmica produzida e a energia térmica dissipada (LEGATES et al., 1991). Um aumento da temperatura retal significa que o animal está estocando calor, e se este não esta dissipando, o estresse calórico manifesta-se. A temperatura retal média de ovinos é $39,1^{\circ} \mathrm{C}$ segundo Swenson \& Reece (1996). Todas as temperaturas retais registradas neste experimento foram acima de $39^{\circ} \mathrm{C}$, mas não ultrapassaram o valor de $39,72{ }^{\circ} \mathrm{C}$ registrado para o $1 / 2$ Santa Inês $+1 / 2$ Dorper no turno da tarde. De acordo com Mcdowell et al. (1976), uma elevação $1^{\circ} \mathrm{C}$ na temperatura retal é o bastante para reduzir o desempenho na maioria das espécies de animais domésticos. Como neste experimento não se constatou tal variação considerou-se que todos genótipos em estudo se comportaram bem diante das situações de conforto, pela manhã, e de perigo, à tarde.

Quanto à frequiência respiratória, observou-se interação entre genótipos e turnos. O genótipo meiosangue $1 / 2$ Santa Inês $+1 / 2$ Dorper diferiu $(\mathrm{P}<0,05)$ de todos os outros genótipos nos turnos da manhã e da tarde, apresentando maior FR, em ambos, respectivamente, 83,33 e 115 movimentos/minuto. O Morada Nova puro diferiu estatisticamente $(\mathrm{P}<0,05)$, pela manhã apenas do mestiço $1 / 2$ Santa Inês x $1 / 2$ Dorper e à tarde dos demais; apresentando menores valores de freqüência respiratória, respectivamente, 43,33 e 56 movimentos/minuto. Os demais genótipos não diferiram entre si $(\mathrm{P}>0,05)$ tanto pela manhã quanto pela tarde. Em relação aos turnos, a variação dos valores obtidos revelou significância $(\mathrm{P}<0,05)$ para todos os genótipos, exceto para o Morada Nova.

Swenson \& Reece (1996) reportam que a freqüência respiratória (FR) média dos ovinos é em torno de 16 a 34 mov/min (movimentos/minuto), valores esses inferiores às médias obtidas neste trabalho. A FR em ruminantes, em ambientes termoneutros oscila entre 24 a $36 \mathrm{mov} / \mathrm{min}$ e acima da temperatura crítica superior $\left(40^{\circ} \mathrm{C}\right)$ esses valores podem estar várias vezes aumentados (SILVA SOBRINHO, 1997; STOBER, 1993). Hales \& Brown (1974) reportam que a taxa de respiração basal da espécie ovina é cerca de 25 a $30 \mathrm{mov} / \mathrm{min}$ (movimentos/minuto), podendo subir, segundo Terrill \& Slee (1991), a $300 \mathrm{mov} / \mathrm{min}$ em ovinos estressados.

Silanikove (2000) relata que a freqüência respiratória pode quantificar a severidade do estresse pelo calor, em que uma freqüência de 40-60, 60-80, 80-120 mov/ min caracteriza um estresse baixo, médio-alto e alto para os ruminantes, respectivamente; e acima de 150 para bovinos e 200 para ovinos, o estresse é classificado como severo. Altas freqüências respiratórias, não significam necessariamente que o animal está em estresse térmico, pois a freqüência respiratória é mais um parâmetro de termorregulação do que um indício de estresse térmico, ou seja, se uma freqüência respiratória estiver alta, mas o animal foi suficiente em eliminar calor, mantendo a homeotermia, pode não ocorrer estresse calórico (BERBIGIER, 1989). Sendo isto variável de ambiente para ambiente, dependendo da eficácia dos mecanismos de calor sensível (condução, convecção e radiação), pois se estes não são eficazes, o organismo animal utiliza mecanismos de dissipação de calor insensível (como a sudorese e/ou freqüência respiratória), para dissipar calor, para regulação homeotérmica. Se os mecanismos evaporativos não foram eficazes, a temperatura retal aumenta consideravelmente, caracterizando o estresse calórico, o que não aconteceu neste experimento. Todavia, Mcdowell (1972) diz que a respiração acelerada e contínua por várias horas pode interferir na ingestão de alimentos, ruminação, podendo afetar o desempenho do animal.

Para freqüência cardíaca não houve efeito significativo $(\mathrm{P}>0,05)$ entre genótipos no turno da manhã, variando de 122 a 133 batimentos/minuto. À tarde os mestiços não diferiram entre si; assim como os puros (P>0,05), variando de 124 a 141 batimentos/minuto. Não ocorreu diferença significativa $(\mathrm{P}>0,05)$ entre turnos.

As médias do gradiente entre temperatura retal e temperatura superficial e do gradiente entre temperatura superficial e temperatura ambiente estão na Tabela 3.

$\mathrm{O}$ gradiente entre as temperaturas retal e superficial revelou significância entre genótipos e turno. Pela manhã, não se verificou significância $(\mathrm{P}>0,05)$ entre os genótipos puros de origem (SI e MN) e os mestiços $1 / 2$ Morada Nova $+1 / 2$ Santa Inês, $1 / 2$ Morada Nova $+1 / 2$ Dorper, entretanto o $1 / 2$ Santa Inês $+1 / 2$ Dorper demonstrou significância $(\mathrm{P}<0,05)$, apresentando maiores valores de gradiente, em relação aos puros e não diferiu estatisticamente $(\mathrm{P}>0,05)$ dos mestiços. À tarde os puros não diferiram estatisticamente $(\mathrm{P}>0,05)$ dos mestiços $1 / 2$ Morada Nova X 1/2 Dorper e 1/2 Morada Nova + 1/2 Santa Inês, mas o 1/2 Santa Inês $+1 / 2$ Dorper demonstrou significância $(\mathrm{P}<0,05)$, apresentando maiores valores de gradiente, em relação aos demais genótipos, exceto o $1 / 2$ Morada Nova $+1 / 2$ Dorper. O puro sangue Morada Nova apresentou menor gradiente pela manhã e, à tarde, não diferiu estatisticamente dos genótipos que apresentaram menor gradiente (Santa Inês e 1/2 Morada Nova + 1/2 Santa Inês). Houve efeito significativo $(\mathrm{P}<0,05)$ para variação entre turnos, em relação ao gradiente entre temperatura retal e superficial, para todos os genótipos. 
TABELA 2 - Médias da temperatura retal (TR), freqüência respiratória (FR) e freqüência cardíaca (FC) de ovinos Santa Inês (SI), Morada Nova (MN), 1/2 Santa Inês + 1/2 Dorper (SI X Dr), 1/2 Morada Nova + 1/2 Dorper (MN X Dr) e 1/2 Morada Nova + 1/2 Santa Inês (MN X SI) em função do genótipo e do turno.

\begin{tabular}{lcccccc}
\hline \multirow{2}{*}{ Genótipos } & \multicolumn{2}{c}{ TR $\left({ }^{\circ} \mathbf{C}\right)$} & \multicolumn{2}{c}{ FC (bat/min) } & \multicolumn{2}{c}{ FR (mov/min) } \\
\cline { 2 - 7 } & Manhã & Tarde & Manhã & Tarde & Manhã & Tarde \\
\hline SI & $39,21^{\mathrm{Aab}}$ & $39,46^{\mathrm{Aab}}$ & $122^{\mathrm{Aa}}$ & $124^{\mathrm{Aa}}$ & $57,66^{\mathrm{Aa}}$ & $85^{\mathrm{Bb}}$ \\
MN & $39,02^{\mathrm{Aa}}$ & $39,24^{\mathrm{Aa}}$ & $123^{\mathrm{Aa}}$ & $130^{\mathrm{Aab}}$ & $47,33^{\mathrm{Aa}}$ & $56^{\mathrm{Aa}}$ \\
SI X Dr & $39,66^{\mathrm{Ac}}$ & $39,72^{\mathrm{Ac}}$ & $133^{\mathrm{Aa}}$ & $141^{\mathrm{Ab}}$ & $83,33^{\mathrm{Ab}}$ & $115^{\mathrm{Bc}}$ \\
MN X Dr & $39,26^{\mathrm{Aab}}$ & $39,54^{\mathrm{Bbc}}$ & $130^{\mathrm{Aa}}$ & $139^{\mathrm{Ab}}$ & $56,83^{\mathrm{Aa}}$ & $89,66^{\mathrm{Bb}}$ \\
MN X SI & $39,38^{\mathrm{Ab}}$ & $39,59^{\mathrm{Abc}}$ & $124^{\mathrm{Aa}}$ & $132^{\mathrm{Aab}}$ & $50,5^{\mathrm{Aa}}$ & $91,5^{\mathrm{Bb}}$ \\
\hline \multicolumn{1}{c}{ CV $(\%)$} & \multicolumn{3}{c}{7,86} & \multicolumn{3}{c}{23,6}
\end{tabular}

Médias seguidas de letras maiúsculas na linha e minúsculas na coluna diferentes são estatisticamente diferentes $(\mathrm{P}<0,05)$ dentro de cada parâmetro, pelo teste de Tukey $5 \%$.

TABELA 3 - Médias do gradiente ("TRTS) entre temperatura retal (TR) e superficial (TS) e do gradiente ("TSTA) entre temperaturas superficial (TS) e do ambiente (TA) de ovinos Santa Inês (SI), Morada Nova (MN) e seus mestiços entre SI e MN e com a Dorper (Dr) em função do genótipo e turno.

\begin{tabular}{lcccc}
\hline \multirow{2}{*}{ Genótipos } & \multicolumn{2}{c}{$\Delta$ TRTS $\left({ }^{\circ} \mathbf{C}\right)$} & \multicolumn{2}{c}{$\Delta$ TSTA $\left({ }^{\circ} \mathbf{C}\right)$} \\
\cline { 2 - 5 } & Manhã & Tarde & $10,83^{\mathrm{Aa}}$ & Tarde \\
\hline SI & $8,48^{\mathrm{Aa}}$ & $6,73^{\mathrm{Ba}}$ & $10,74^{\mathrm{Aa}}$ & $3,43^{\mathrm{Ba}}$ \\
MN & $8,38^{\mathrm{Aa}}$ & $7,04^{\mathrm{Bab}}$ & $10,12^{\mathrm{Aa}}$ & $2,88^{\mathrm{Bc}}$ \\
SI X Dr & $9,58^{\mathrm{Ab}}$ & $8,54^{\mathrm{Bc}}$ & $10,29^{\mathrm{Aa}}$ & $3,23^{\mathrm{Bbc}}$ \\
MN X Dr & $9,08^{\mathrm{Aab}}$ & $8,01^{\mathrm{Bbc}}$ & $10,77^{\mathrm{Aa}}$ & $4,57^{\mathrm{Bab}}$ \\
MN X SI & $8,71^{\mathrm{Aab}}$ & $6,73^{\mathrm{Ba}}$ & \multicolumn{3}{c}{10,54} \\
\hline \multicolumn{1}{c}{ CV $(\%)$} & & & \multicolumn{3}{c}{} \\
\hline
\end{tabular}

Médias seguidas de letras maiúsculas na linha e minúsculas na coluna diferentes são estatisticamente diferentes $(\mathrm{P}<0,05)$ dentro de cada parâmetro, pelo teste de Tukey $5 \%$.

Para o gradiente térmico entre temperatura superficial e temperatura do ambiente houve interação entre genótipos e turnos. Pela manhã, não houve diferença estatística $(\mathrm{P}>0,05)$ entre os genótipos. À tarde $\mathrm{o} 1 \frac{1}{2}$ Santa Inês $+1 / 2$ Dorper diferiu estatisticamente $(\mathrm{P}<0,05)$ dos demais genótipos, exceto do mestiço $1 / 2$ Morada Nova $+1 / 2$ Dorper, apresentando menor gradiente. Houve significância $(\mathrm{P}<0,05)$ entre turnos para todos os genótipos. A existência de gradiente entre a superfície do animal e o meio é um fator importante para dissipação de calor pelos mecanismos não evaporativos (condução, convecção e radiação). Pode se perceber que os gradientes à tarde sempre foram menores que pela manhã, isto em função da temperatura do ar ser mais alta à tarde, fator que dificulta o processo de dissipação de calor.
Áreas quentes e úmidas representam problemas para o desempenho animal (LEVA, 1998), pois dificultam a dissipação de calor pelo gradiente baixo entre as temperaturas superficial e a ambiental. Assim, explicam-se as altas temperaturas retais, pelo acúmulo de calor endógeno e as altas frequiências respiratórias como um mecanismo de termorregulação.

\section{CONCLUSÃO}

Os ovinos das raças Santa Inês, Morada Nova e seus mestiços com a raça Dorper apresentam alta capacidade fisiológica para manter a homeotermia em ambiente quente.

Os ovinos das raças Santa Inês e Morada Nova e seus mestiços com a raça Dorper apresentam alto grau de adaptabilidade, às condições Semi-áridas do Brasil. 


\section{REFERÊNCIAS BIBLIOGRÁFICAS}

ABI-SAAB, S.; SLEIMAN, F. T. Physiological responsses to stress of filial crosses compared to local Awassi sheep. Small Ruminant Research, Amsterdam, v. 6, p. 55-59, 1995.

AGRICULTURAL AND FOOD RESEARCH COUNCIL. Energy and protein requirements of ruminants. Washington: CAB International, 1995. 159 p.

AGRICULTURAL RESEARCH COUNCIL. The nutrient requirements of ruminant livestock. London, 1980.351 p.

BACCARI JÚNIOR, F. Métodos e técnicas de avaliação da adaptabilidade dos animais às condições tropicais. In: SIMPÓSIO INTERNACIONAL DE BIOCLIMATOLOGIA ANIMAL NOS TRÓPICOS: PEQUENOS E GRANDES RUMINANTES, 1., 1990, Sobral, CE. Anais... Sobral: Embrapa-CNPC, 1990. p. 9-17.

BAÊTA, F. C.; SOUZA, C. F. Ambiência em edificações rurais: conforto animal. Viçosa: UFV, 1997. 246 p.

BERBIGIER, P. Effect of heat on intensive meat production in the tropics: cattle, sheep and goat, pigs. In: CICLO INTERNACIONAL DE PALESTRAS SOBRE BIOCLIMATOLOGIA ANIMAL, 1., 1989, Botucatu. Anais... Jaboticabal: FMVZ/UNESP/FUNEP, 1989. p. 7-44.

BIANCA, W.; KUNZ, P. Physiological reactions of three breedes of goats to cold, heat and hight altitude. Livestock Production Science, Amsterdam, v. 5, n. 1, p. 57-69, 1978.

BRASIL. Secretaria Nacional de Irrigação. Departamento Nacional de Meteorologia. Normas climatológicas: 19611990. Brasília, DF: Embrapa-SPI, 1992. 84 p.

BROWN-BRANDL, T. M.; NIENABER, J. A.; EIGENBERG, R. A. Thermoregulatory responses of feeder cattle. Journal of Thermal Biology, [S.1.], v. 28, p. 149-157, 2003.

BUFFINGTON, D. E.; COLLAZZO-AROCHO, A.; CANTON, G. H. Black globe- humidity index (BGHI) as comfort equation for dairy cows. Transaction of the ASAE, [S.1.], v. 24, p. 711-714, 1981.

CHIMINEAU, P. Médio ambiente y reproduccion animal. World Animal Review, Roma, v. 77, n. 1, p. 2-14, 1993.
DOMINGUES, O. Introdução à zootecnia. 3. ed. Rio de Janeiro: SIA, 1968. 392 p.

HABEEB, A. L. M.; MARAY, I. F. M.; KAMAL, T. H. Farm animals and the environment. Cambridge: $\mathrm{CAB}$, 1992. $428 \mathrm{p}$.

HALES, J. R. S.; BROWN, G. D. Net energetic and thermoregulatory efficiency during panting in the sheep. Comp. Biochemical Physiology, [S.1.], v. 49, p. 413-422, 1974.

HARDY, R. N. Temperatura e vida animal. São Paulo: EPU, $1981.91 \mathrm{p}$.

HARRIS, D. L.; SHRODE, R. R.; RUPEL, I. W. Study of solar radiation as related to physiological and production responses of Lactating Holstein and Jersey cows. Journal of Dairy Science, Champaign, v. 43, n. 9, p. 1255-1262, Sept. 1960.

LEGATES, J. E.; FARTHING, B. R.; CASADY, R. B. Body temperature and respiratory rate of lactating dairy cattle under field and chamber conditions. Journal Dairy Science, Champaign, v. 74, p. 2491-2500, 1991.

LEVA, P. Impacto ambiental en la producción lechera en la Cuenca Central Argentina. In: CONGRESSO BRASILEIRO DE BIOMETEOROLOGIA, 2., 1998, Goiânia. Anais... Goiânia: [s.n.], 1998. p. 120-136.

McDOWELL, R. E. Improvement of livestock production in warm climates. San Francisco: Freeman, 1972. 711 p.

McDOWELL, R. E.; HOOVEN, N. W.; CAMOENS, J. K. Effects of climate onperformance of Holsteins in first lactation. Journal of Dairy Science, Champaign, v. 59, p. 965-973, 1976.

MONTY JUNIOR, D. E.; KELLY, L. M.; RICE, W. R. Aclimatization of St Coix, Karakul and Rambouillet sheep to intense and dry summer heat. Small Ruminant Research, [S.1.], v. 4, n. 4, p. 379-392, 1991.

OLIVIER, J. J. Breeding plans for Dorper sheep and Boer gotas in South Africa. In: SIMPÓSIO INTERNACIONAL SOBRE CAPRINOS E OVINOS DE CORTE, 1., 2000, João Pessoa, PB. Anais... João Pessoa: EMEPA-PB, 2000. p. 213-230. 
SAS INSTITUTE. Statistical analysis System: user's guide: statistics. Version 6.11. Cary, 1996. 842 p.

SHORODE, R. R.; QUAZI, F. R.; RUPEL, I. W. Variation in rectal temperature, respiration rate, and pulse rate of cattle as related to variation in four environmental variables. Journal of Dairy Science, Champaign, v. 43, n. 9, p. 12351244, Sept. 1960.

SILANIKOVE, N. Effects of heat stress on the welfare of extensively managed domestic ruminants. Livestock Production Science, [S.1.], v. 67, p. 1-18, 2000.

SILVA, R. G.; GONDIM, A. G. Comparação entre as raças Sindi e Jersey e seus mestiços, relativamente à tolerância ao calor na região Amazônica. Pesquisa Agropecuária Brasileira, Brasília, v. 6, p. 37-44, 1971.

SILVA SOBRINO, A. G. Criação de ovinos. Jaboticabal: Funep, 1997. 203 p.
SOUZA, B. B.; SILVA, A. M.; VIRGINIO, R. S.; GUEDES JÚNIOR, D. B.; AMORIM, F. U. Comportamento fisiológico de ovinos deslanados no semi-árido expostos em ambiente sol e em ambiente sombra. Veterinária e Zootecnia, São Paulo, v. 2, p. 1-8, 1990.

STOBER, M. Identificação,anamnese, regras básicas da técnica do exame clínico geral. In: . Exame clínico dos bovinos. 3. ed. Rio de Janeiro: Guanabara Koogan, 1993. $419 \mathrm{p}$.

SWENSON, M. J.; REECE, W. O. Dukes fisiologia dos animais domésticos. 11. ed. Rio de Janeiro: Guanabara Koogan, 1996. 855 p.

TERRILL, C. E.; SLEE, J. Breed differences in adaptation of sheep. In: MAIJALA, K. Genetic

resources of pigs, sheep and goat. Amsterdam: Elsevier, 1991. p. 195-233. 